\title{
Risk Factors for Metachronous Gastric Neoplasms in Patients Who Underwent Endoscopic Resection of a Gastric Neoplasm
}

\author{
Hyuk Yoon*, Nayoung Kim* ${ }^{*}{ }^{\dagger}$, Cheol Min Shin*, Hye Seung Lee ${ }^{\ddagger}$, Bo Kyoung Kim ${ }^{\dagger}$, Gyeong Hoon Kang ${ }^{\S}$, Jung Mogg Kim", \\ Joo Sung $\mathrm{Kim}^{\dagger}$, Dong Ho Lee ${ }^{{ }^{\dagger}}$, and Hyun Chae Jung ${ }^{\dagger}$ \\ ${ }^{*}$ Department of Internal Medicine, Seoul National University Bundang Hospital, Seongnam, ${ }^{\dagger}$ Department of Internal Medicine and Liver \\ Research Institute, Seoul National University College of Medicine, Seoul, ${ }^{\ddagger}$ Department of Pathology, Seoul National University Bundang \\ Hospital, Seongnam, ${ }^{s}$ Department of Pathology, Laboratory of Epigenetics, Cancer Research Institute, Seoul National University College of \\ Medicine, and "Department of Microbiology, Hanyang University College of Medicine, Seoul, Korea
}

Background/Aims: To identify the risk factors for metachronous gastric neoplasms in patients who underwent an endoscopic resection of a gastric neoplasm. Methods: We prospectively collected clinicopathologic data and measured the methylation levels of HAND1, THBD, APC, and MOS in the gastric mucosa by methylation-specific real-time polymerase chain reaction in patients who underwent endoscopic resection of gastric neoplasms. Results: A total of 257 patients with gastric neoplasms (113 low-grade dysplasias, 25 highgrade dysplasias, and 119 early gastric cancers) were enrolled. Metachronous gastric neoplasm developed in $7.4 \%$ of patients during a mean follow-up of 52 months. The 5-year cumulative incidence of metachronous gastric neoplasm was 4.8\%. Multivariate analysis showed that moderate/severe corpus intestinal metaplasia and family history of gastric cancer were independent risk factors for metachronous gastric neoplasm development; the hazard ratios were 4.12 (95\% confidence interval [Cl], 1.23 to $13.87 ; \mathrm{p}=0.022$ ) and 3.52 (95\% Cl, 1.09 to $11.40 ; p=0.036$ ), respectively. The methylation level of MOS was significantly elevated in patients with metachronous gastric neoplasms compared age- and sexmatched patients without metachronous gastric neoplasms $(p=0.020)$. Conclusions: In patients who underwent endoscopic resection of gastric neoplasms, moderate/severe corpus intestinal metaplasia and a family history of gastric cancer were independent risk factors for metachronous gastric neoplasm, and MOS was significantly hypermethylated in patients with metachronous gastric neoplasms. (Gut Liver 2016;10:228-236)
Key Words: Stomach neoplasms; Metastasis; Risk factors; Therapeutics

\section{INTRODUCTION}

Metachronous gastric cancer (MGC) develops in a considerable portion of patients who underwent endoscopic resection (ER) of early gastric cancer (EGC). ${ }^{1,2}$ Therefore, it is very important to elucidate risk factors for MGC in these patients to establish an appropriate surveillance strategy. Old age, family history of gastric cancer, extensive corpus atrophy, intestinal metaplasia (IM), and persistent Helicobacter pylori infection were suggested as risk factors for MGC in previous studies..$^{3-5}$ On the other hand, although the optimal treatment strategy has not yet been established, aggressive treatments such as endoscopic mucosal resection or endoscopic submucosal dissection have been more frequently performed for gastric dysplasia. The reason is that gastric dysplasia is a more advanced premalignant lesion than gastric atrophy/IM; additionally it is focal lesion which makes it easy to try preemptive ER in contrast to gastric atrophy/IM. Therefore, it would be practical to manage EGC and gastric dysplasia in conjunction, as "gastric neoplasm," although the interval of surveillance after ER could vary based on whether the lesion is cancer or dysplasia. However, few studies have evaluated risk factors for metachronous gastric neoplasm (MGN) including dysplasia, in the patients who undergo ER of gastric neoplasm.

Gastric cancer develops through the accumulation of genetic and epigenetic alterations. Recently, attention has focused on aberrant DNA methylation as an important mechanism of gastric carcinogenesis. H. pylori infection induces chronic

Correspondence to: Nayoung Kim

Department of Internal Medicine, Seoul National University Bundang Hospital, Seoul National University College of Medicine, 82 Gumi-ro 173beon-gil, Bundang-gu, Seongnam 463-707, Korea

Tel: +82-31-787-7008, Fax: +82-31-787-4051, E-mail: nayoungkim49@empas.com

Received on December 1, 2014. Revised on January 25, 2015. Accepted on January 25, 2015. Published online June 19, 2015

pISSN 1976-2283 eISSN 2005-1212 http://dx.doi.org/10.5009/gnl14472

(a) This is an Open Access article distributed under the terms of the Creative Commons Attribution Non-Commercial License (http://creativecommons.org/licenses/by-nc/4.0) which permits unrestricted non-commercial use, distribution, and reproduction in any medium, provided the original work is properly cited. 
inflammation, increased secretion of several cytokines and hypermethylation of promoter regions of tumor suppressor genes. Consequently, tumor suppressor genes are accumulatively inactivated, resulting in the development of gastric cancer. This is a well-known the concept of field cancerization. ${ }^{6,7}$ That is, by the time gastric cancer becomes visible, the stomach likely harbors areas containing premalignant lesions. ${ }^{8}$ Therefore, we could expect that the higher the aberrant DNA methylation related to gastric carcinogenesis in a patient who underwent ER of gastric neoplasm, the higher the risk of MGN due to field cancerization. However, there are few studies on this topic. IM is one of the strongest risk factors for gastric cancer ${ }^{9}$ and it is considered as the key link in the process from $H$. pylori infection to gastric cancer through the aberrant DNA methylation. We have recently elucidated THBD, HAND1, and APC as hypermethylated genes related to IM. ${ }^{10}$ Genome-wide DNA methylation profiles in noncancerous gastric mucosae have identified MOS as a hypermethylated gene in the gastric cancer irrespective of H. pylori infection. ${ }^{11}$ In subsequent studies, we found that the methylation level of MOS correlated with severity of IM. ${ }^{12,13}$ We therefore speculated that THBD, HAND1, APC, and MOS which are related to severity of IM and show persistent methylation after $H$. pylori eradication could be molecular risk factors for MGN.

The aim of the current study was to identify risk factors for MGN among diverse clinicopathologic factors and above-mentioned hypermethylated genes in the patients who underwent ER of gastric neoplasm.

\section{MATERIALS AND METHODS}

\section{Patients}

Between October 2004 and July 2013, patients diagnosed with gastric neoplasm by endoscopic biopsy who underwent ER by one experienced endoscopist (N.K.) were prospectively enrolled at Seoul National University Bundang Hospital, Seongnam, South Korea. All participants were ethnically Korean. From this subject pool, only patients who had been followed up by regular endoscopy for more than 12 months were enrolled in the study. Patients were excluded from this study based on the following criteria: (1) patients whose final diagnosis was beyond expanded criteria of endoscopic submucosal dissection for $\mathrm{EGC}^{14}$ on pathologic review of the resected specimen; and (2) patients who had another underlying cancer. This study was approved by the Institutional Review Board of Seoul National University Bundang Hospital (IRB number: B-1403-242-302).

\section{Determination of $\boldsymbol{H}$. pylori infection status}

To determine $H$. pylori infection status, three biopsy-based tests (histology, rapid urease test, and culture) were used. A total of 10 biopsy specimens were taken from the gastric mucosa of each patient. Among these 10 specimens, four were used for histological evaluation of $H$. pylori infection by modified Giemsa staining (one each from the greater and lesser curvature of the antrum and body). Another four specimens from the four gastric mucosa areas mentioned above were used for $H$. pylori culturing. The remaining two specimens from the lesser curvature of the antrum and body were used for the rapid urease test (CLOtest; Delta West, Bentley, Australia). The protocols for these three biopsy-based tests have been described in detail. ${ }^{15}$ Current $H$. pylori infection was defined as positive from any of these three tests.

Two other methods were used to identify patients who had a previous $H$. pylori infection. First, an enzyme-linked immunosorbent assay (ELISA) was used to screen for immunoglobulin G (IgG) specific for $H$. pylori in each patient's serum (Genedia H. pylori ELISA; Green Cross Medical Science Corp., Eumsung, Korea). Second, each patient was questioned about his history of $H$. pylori eradication. If the patient had a history of $H$. pylori eradication or $H$. pylori serology was positive but no bacteria were found by histology, the rapid urease test, or culturing, the patient was diagnosed with a past $H$. pylori infection without current ongoing infection. ${ }^{16}$

\section{Evaluation of gastric atrophy and IM}

The severity of gastric atrophy in each patient was evaluated by the serum pepsinogen (PG) test. Fasting serum was collected from the patients, and serum concentrations of PG I and II were measured using a Latex-enhanced Turbidimetic Immunoassay (Shima Laboratories, Tokyo, Japan). Based on the results of the serum PG tests, the patients were categorized as having no, mild to moderate, or severe gastric atrophy according to the definition of Miki et al. ${ }^{17}$ No atrophy was defined as PG I $>70$ and PG $\mathrm{I} / \mathrm{II}$ ratio $>3.0$. Severe atrophy was defined as PG I $\leq 30$ and PG $\mathrm{I} / \mathrm{II} \leq 2.0$. All other patients were identified with mild to moderate atrophy.

To evaluate the severity of IM, we recorded the updated Sydney system scores of the four biopsy specimens from each patient used for histological evaluation of $H$. pylori infection. ${ }^{18}$

\section{ER and follow-up}

Each patient underwent one of two types of ER. Endoscopic mucosal resection was performed usually for the small gastric dysplasia. For dysplasia larger than $2 \mathrm{~cm}$ and most EGCs, endoscopic submucosal dissection was preferred. The technical methods of endoscopic mucosal resection and endoscopic submucosal dissection were previously described. ${ }^{19}$

During the follow-up period, endoscopy was performed routinely at 3, 6, and 12 months, and then annually to assess the completeness of resection as well as to detect metachronous lesions. Biopsy samples were taken from the scar of ER or other suspicious mucosal abnormalities. Abdominal computed tomography and chest radiography were performed annually to assess distant metastases. MGN was defined as gastric dysplasia or 
cancer which was developed at least 12 months after initial ER of gastric neoplasm.

\section{Quantitative methylation-specific polymerase chain reaction}

Patients with and without MGN among the included subjects were matched for sex and age by 1:1. In these patients, methylation-specific polymerase chain reaction (PCR) was performed and the methylation level of each gene was compared between the two groups. The protocols for DNA preparation and quantitative methylation-specific PCR have been described in detail. ${ }^{10}$ Briefly, genomic DNA was extracted directly from nonneoplastic antral biopsy specimens. After isolation, the DNA was subjected to sodium bisulfite modification. ${ }^{20}$ DNA methylation in the four selected CpG sites (HAND1, THBD, APC, and MOS) were evaluated as previously. ${ }^{10,11}$ The primer sequences for methylationspecific PCR were designed using BLAST (http://www.ncbi.nlm. nih.gov/BLAST/). An aliquot of $2 \mu \mathrm{L}$ was used for real-time PCR with a primer set specific to methylated or unmethylated sequences, with a specific annealing temperature of $53^{\circ} \mathrm{C}$ to $66^{\circ} \mathrm{C}$. Real-time PCR was performed using $23 S_{Y B R V}{ }^{\circledR}$ Premix Ex Taq $^{\text {TM }}$ (Takara Bio, Shiga, Japan) and ABI PRISM ${ }^{\circledR} 7000$ Sequence Detection System (Applied Biosystems, Foster, CA, USA). Standard DNA was prepared by cloning PCR products into the pGEM-T Easy vector (Promega, Madison, WI, USA). The number of molecules in a test sample was determined by comparing the amplification with those of standard samples containing a known number of molecules $\left(10^{6}-10^{1}\right)$. The number of methylated and unmethylated molecules was measured separately, and the methylation level was calculated as following: methylation level=number of methylated molecules/total number of DNA molecules (methylated+unmethylated molecules).

\section{Statistical analysis}

SPSS for Windows version 18.0 (SPSS Inc., Chicago, IL, USA) was used for the statistical analysis. Baseline clinicopathologic characteristics of the patients were presented as descriptive data. Cumulative probabilities of MGN were estimated by the KaplanMeier method. The log-rank test was used to compare the timeto-event curves of MGN according to the severity of IM in the gastric body. A univariate Cox proportional hazards model was used to identify possible covariates as significant risk factors for MGN. Then, the variables with $\mathrm{p}<0.05$ were subjected to multivariate Cox proportional hazards model to identify independent contribution. In addition, the variables which were considered to be possible risk factors for MGN based on the previous studies were also analyzed in multivariate model. The methylation level of four genes in patients with and without MGN were compared by Mann-Whitney U test, as the data from the two groups were not normally distributed. All results were considered statistically significant when $\mathrm{p}$-values were less than 0.05 .
Table 1. Baseline Clinicopathologic Characteristics of the 257 Patients Who Underwent Endoscopic Resection of Gastric Neoplasms

\begin{tabular}{|c|c|}
\hline Characteristic & Value \\
\hline Age, yr & $61.8 \pm 9.4$ \\
\hline \multicolumn{2}{|l|}{ Sex } \\
\hline Male & $183(71.2)$ \\
\hline Female & $74(28.8)$ \\
\hline \multicolumn{2}{|l|}{ Family history of gastric cancer* } \\
\hline No & $199(77.4)$ \\
\hline Yes & $50(19.5)$ \\
\hline \multicolumn{2}{|l|}{ Smoking status } \\
\hline Never & $102(39.7)$ \\
\hline Past & $113(44.0)$ \\
\hline Current & $42(16.3)$ \\
\hline \multicolumn{2}{|l|}{ H. pylori infection status } \\
\hline Never & $30(11.7)$ \\
\hline Past & $69(26.8)$ \\
\hline Current & $158(61.5)$ \\
\hline \multicolumn{2}{|c|}{ Gastric atrophy by serum pepsinogen test* } \\
\hline Not severe & $209(81.3)$ \\
\hline Severe $^{\dagger}$ & $44(17.1)$ \\
\hline \multicolumn{2}{|l|}{ IM (antrum) } \\
\hline Absent or mild & $125(48.6)$ \\
\hline Moderate or severe & $132(51.4)$ \\
\hline \multicolumn{2}{|l|}{ IM (body) } \\
\hline Absent or mild & $188(73.2)$ \\
\hline Moderate or severe & $69(26.8)$ \\
\hline \multicolumn{2}{|l|}{ Type of gastric neoplasm } \\
\hline Low-grade dysplasia & $113(44.0)$ \\
\hline High-grade dysplasia & $25(9.7)$ \\
\hline Early gastric cancer & $119(46.3)$ \\
\hline \multicolumn{2}{|l|}{ No. of neoplasm } \\
\hline Single & $239(93.0)$ \\
\hline Multiple & $18(7.0)$ \\
\hline \multicolumn{2}{|l|}{ Location of neoplasm } \\
\hline Upper or middle third & $63(24.5)$ \\
\hline Lower third & 194 (75.5) \\
\hline Size of neoplasm, $\mathrm{cm}$ & $1.2 \pm 0.8$ \\
\hline \multicolumn{2}{|l|}{ Type of endoscopic resection } \\
\hline Endoscopic mucosal resection & $164(63.8)$ \\
\hline Endoscopic submucosal dissection & $93(36.2)$ \\
\hline
\end{tabular}

Data are presented as mean \pm SD or number (\%).

H. pylori, Helicobacter pylori; IM, intestinal metaplasia.

*The data regarding the family history of gastric cancer were missing in eight patients, and the serum pepsinogen test was not performed in four patients; ${ }^{\dagger}$ Severe atrophy was defined as pepsinogen I $\leq 30$ and pepsinogen $\mathrm{I} / \mathrm{II} \leq 2.0$. 


\section{RESULTS}

A total of 257 patients who underwent ER of gastric neoplasm were enrolled. Baseline clinicopathologic characteristics of the patients were shown in Table 1. Low-grade dysplasia, high-grade dysplasia, and EGC were 44.0\%, 9.7\%, and 46.3\%, respectively. The mean follow-up period was $52 \pm 29$ months and mean $5.0 \pm 2.5$ times of surveillance endoscopy were performed in each patient during the follow-up period. MGN developed in 19 patients (7.4\%). Clinicopathologic characteristics of MGNs

Table 2. Clinicopathologic Characteristics of 19 Metachronous Gastric Neoplasm in Patients Who Underwent Endoscopic Resection of Gastric Neoplasms

\begin{tabular}{lc}
\hline \multicolumn{1}{c}{ Characteristic } & No. (\%) \\
\hline Type of MGN & $10(52.6)$ \\
Low-grade dysplasia & $2(10.5)$ \\
High-grade dysplasia & $6(31.6)$ \\
Early gastric cancer & $1(5.3)$ \\
Advanced gastric cancer & \\
Location of MGN & $4(21.1)$ \\
Upper third & $5(26.3)$ \\
Middle third & $10(52.6)$ \\
Lower third & \\
Treatment modality for MGN & $13(68.4)$ \\
Endoscopic mucosal resection & $4(21.1)$ \\
Endoscopic submucosal dissection & $1(5.3)$ \\
Surgery after endoscopic submucosal dissection & $1(5.3)$ \\
Chemotherapy & \\
Second metachronous neoplasm after treatment of MGN* & $15(83.3)$ \\
No & $3(16.7)$ \\
Yes & \\
\hline
\end{tabular}

MGN, metachronous gastric neoplasm.

*One pateint who developed advanced gastric cancer was excluded in this anaylsis. were summarized in Table 2. Among 19 MGNs, the rate of gastric cancer was 36.8\% (7/19). Except one advanced gastric cancer, all MGNs were treated by ER or operation. However, secondary MGN developed in 16.7\% (3/18).

The 5-year cumulative incidence of MGN was 4.8\% (Fig. 1). In the univariate analysis, moderate or severe IM in the gastric body was the only risk factor for MGN (hazard ratio, 3.11; 95\% confidence interval [CI], 1.24 to $7.80 ; \mathrm{p}=0.016$ ) (Table 3). The 5-year cumulative incidence of MGN in the patients with no/ mild corpus IM and in the patients with moderate/severe corpus IM was $1.1 \%$ and $10.8 \%$, respectively. The cumulative probability of MGN was significantly different according to the severity of corpus IM (absent or mild vs moderate or severe, $p=0.011$ by log-rank test) (Fig. 2). In the multivariate Cox proportional hazard model, age, family history of gastric cancer, $H$. pylori infection status, and gastric atrophy by serum PG test were also analyzed with severity of IM, because these variables were suggested as risk factors for MGC in previous studies. The results showed that moderate or severe IM in the gastric body and family history of gastric cancer were independent risk factors for MGN after adjusting for other variables; hazard ratios were

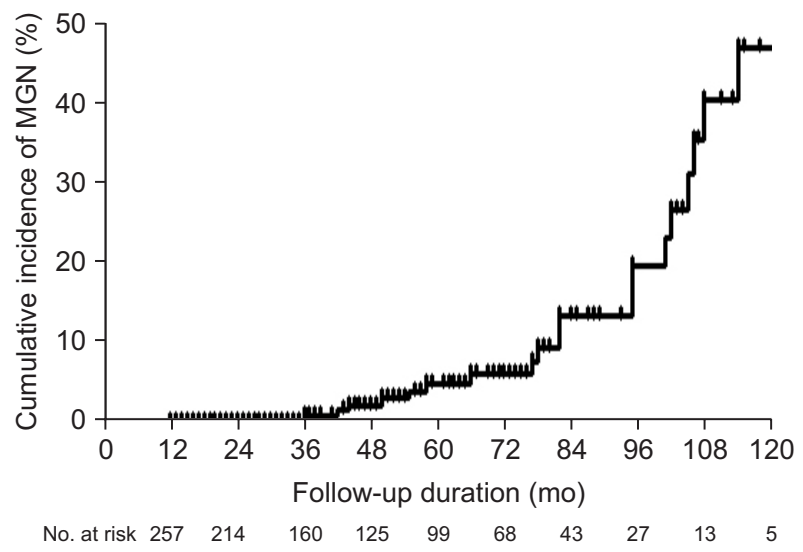

Fig. 1. Kaplan-Meier estimates of the cumulative incidence of metachronous gastric neoplasm (MGN).

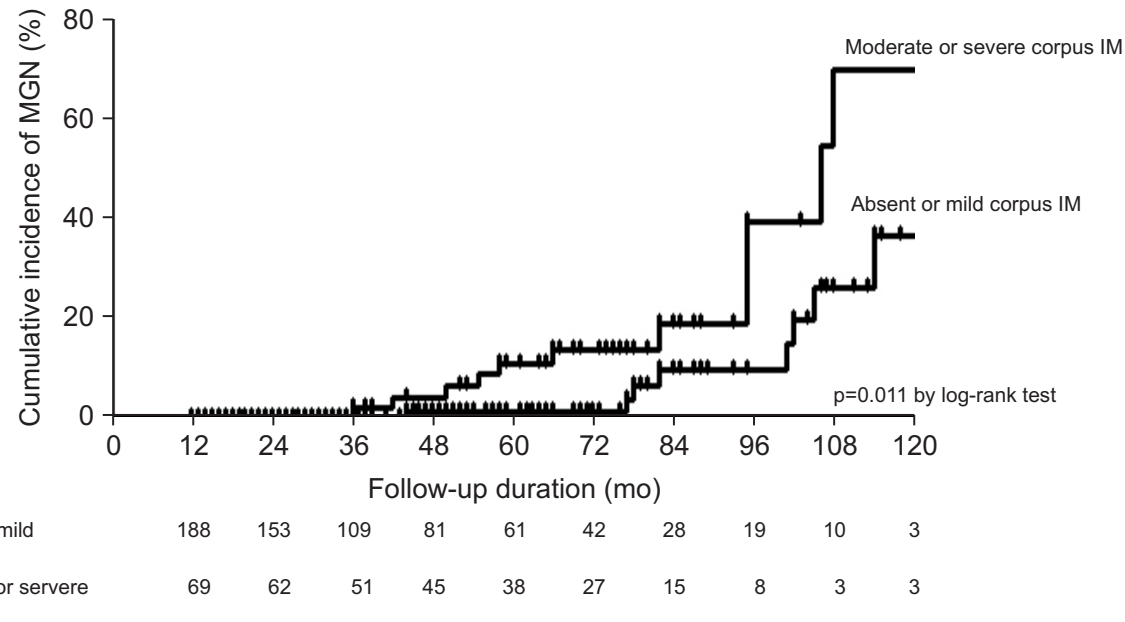

No. at risk

Absent or mild corpus IM

Moderate or servere corpus IM $\begin{array}{llllllllll}69 & 62 & 51 & 45 & 38 & 27 & 15 & 8 & 3 & 3\end{array}$
Fig. 2. Kaplan-Meier estimates of the cumulative incidence of metachronous gastric neoplasm (MGN) according to the severity of intestinal metaplasia (IM) in the gastric body. 
Table 3. Univariate and Multivariate Cox Regression Analysis of the Risk Factors for Metachronous Gastric Neoplasm

\begin{tabular}{|c|c|c|c|c|c|c|}
\hline \multirow{2}{*}{ Variable } & \multirow{2}{*}{ No. } & \multirow{2}{*}{ No. of MGN (\%) } & \multicolumn{2}{|c|}{ Univariate analysis } & \multicolumn{2}{|c|}{ Multivariate analysis } \\
\hline & & & HR $(95 \%$ CI $)$ & p-value & $\mathrm{HR}(95 \% \mathrm{CI})$ & p-value \\
\hline \multicolumn{7}{|l|}{ Sex } \\
\hline Female & 74 & $3(4.1)$ & 1 & & - & \\
\hline Male & 183 & $16(8.7)$ & $1.99(0.58-6.88)$ & 0.275 & - & - \\
\hline \multicolumn{7}{|l|}{ Age, yr } \\
\hline$<65$ & 99 & $11(7.9)$ & 1 & & 1 & \\
\hline$\geq 65$ & 158 & $8(6.8)$ & $1.29(0.52-3.22)$ & 0.589 & $1.22(0.45-3.31)$ & 0.699 \\
\hline \multicolumn{7}{|c|}{ Family history of gastric cancer } \\
\hline No & 199 & $12(6.0)$ & 1 & & 1 & \\
\hline Yes & 50 & $6(12.0)$ & $1.58(0.59-4.24)$ & 0.364 & $3.52(1.09-11.40)$ & $0.036^{*}$ \\
\hline \multicolumn{7}{|l|}{ Smoking status } \\
\hline Never & 102 & $7(6.9)$ & 1 & & - & \\
\hline Past & 113 & $7(6.2)$ & $0.84(0.29-2.40)$ & 0.745 & - & - \\
\hline Current & 42 & $5(11.9)$ & $1.90(0.59-6.14)$ & 0.284 & - & - \\
\hline \multicolumn{7}{|l|}{ H. pylori infection status } \\
\hline Never & 30 & $3(10.0)$ & 1 & & 1 & \\
\hline Past & 69 & $3(4.3)$ & $0.51(0.10-2.54)$ & 0.413 & $0.63(0.10-3.86)$ & 0.613 \\
\hline Current & 158 & $13(8.2)$ & $0.95(0.27-3.33)$ & 0.930 & $1.33(0.27-6.45)$ & 0.724 \\
\hline \multicolumn{7}{|l|}{ Gastric atrophy by SPT } \\
\hline Not severe & 209 & $13(6.2)$ & 1 & & 1 & \\
\hline Severe $^{\dagger}$ & 44 & $6(13.6)$ & $2.00(0.76-5.28)$ & 0.161 & $1.77(0.57-5.48)$ & 0.326 \\
\hline \multicolumn{7}{|l|}{ IM (antrum) } \\
\hline Absent or mild & 125 & $8(6.4)$ & 1 & & 1 & \\
\hline Moderate or severe & 132 & $11(8.3)$ & $1.74(0.69-4.34)$ & 0.239 & $0.84(0.28-2.54)$ & 0.761 \\
\hline \multicolumn{7}{|l|}{ IM (body) } \\
\hline Absent or mild & 188 & $8(4.3)$ & 1 & & 1 & \\
\hline Moderate or severe & 69 & $11(15.9)$ & $3.11(1.24-7.80)$ & $0.016^{*}$ & $4.12(1.23-13.87)$ & $0.022^{*}$ \\
\hline \multicolumn{7}{|l|}{ Type of neoplasm } \\
\hline Low-grade dysplasia & 113 & $8(7.1)$ & 1 & & - & \\
\hline High-grade dysplasia & 25 & $3(12.0)$ & $0.84(0.22-3.26)$ & 0.803 & - & \\
\hline Early gastric cancer & 119 & $8(6.7)$ & $0.96(0.25-3.75)$ & 0.951 & - & - \\
\hline \multicolumn{7}{|l|}{ No. of neoplasm } \\
\hline Single & 239 & $17(7.1)$ & 1 & & - & \\
\hline Multiple & 18 & $2(11.1)$ & $0.894(0.20-4.00)$ & 0.883 & - & - \\
\hline \multicolumn{7}{|l|}{ Location of neoplasm } \\
\hline Upper or middle third & 63 & $6(9.5)$ & 1 & & - & \\
\hline Lower third & 194 & $13(6.7)$ & $0.60(0.23-1.58)$ & 0.299 & - & - \\
\hline \multicolumn{7}{|l|}{ Size of neoplasm, cm } \\
\hline$\leq 2$ & 227 & $16(7.0)$ & 1 & & - & \\
\hline$>2$ & 30 & $3(10.0)$ & $2.23(0.63-7.82)$ & 0.212 & - & - \\
\hline
\end{tabular}

MGN, metachronous gastric neoplasm; HR, hazard ratio; CI, confidence interval; H. pylori, Helicobacter pylori; SPT, serum pepsinogen test; IM, intestinal metaplasia.

${ }^{*} \mathrm{p}<0.05$; ${ }^{\dagger}$ Severe atrophy was defined as pepsinogen $\mathrm{I} \leq 30$ and pepsinogen $\mathrm{I} / \mathrm{II} \leq 2.0$. 

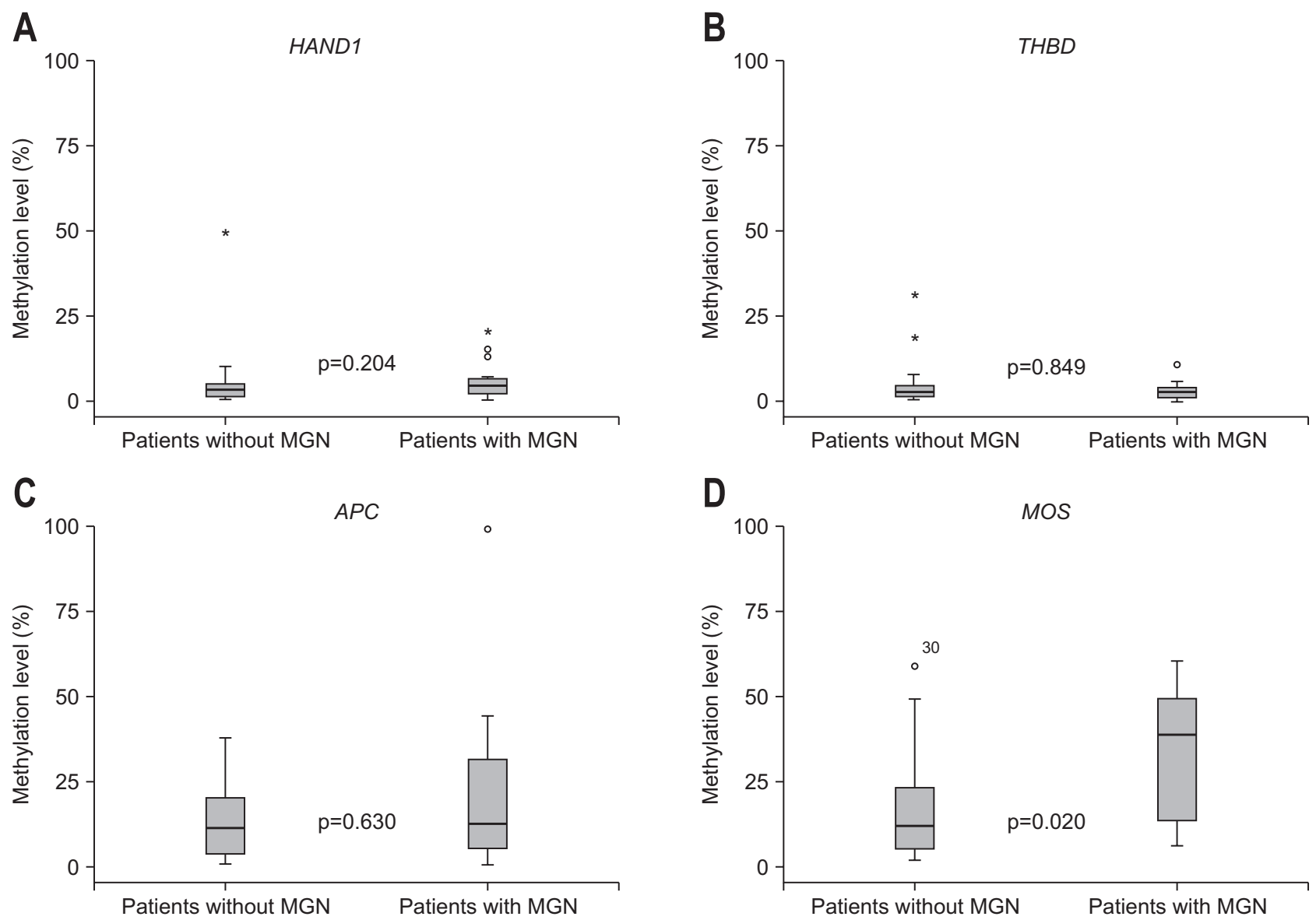

Fig. 3. Methylation levels of the four genes in patients with and without metachronous gastric neoplasm (MGN): (A) HAND1, (B) THBD, (C) APC, and (D) MOS.

4.12 (95\% CI, 1.23 to $13.87 ; \mathrm{p}=0.022)$ and 3.52 (95\% CI, 1.09 to $11.40 ; \mathrm{p}=0.036$ ), respectively (Table 3).

When the methylation levels of HAND1, THBD, APC, and MOS were compared between 19 patients with MGN and 19 age- and sex-matched patients without MGN, by methylation specific RT-PCR, only the methylation level of MOS was significantly elevated in patients with MGN as compared to those without MGN ( $p=0.020$ ) (Fig. 3).

\section{DISCUSSION}

Even though there have been several studies regarding risk factors for MGC after ER of EGC, the methods to evaluate $H$. pylori infection, gastric atrophy and IM were not meticulous and the suggested risk factors varied. In addition, only few studies have included dysplasia which is usually endoscopically resected after detection and needs regular surveillance similar to EGC. Therefore, we attempted to elucidate risk factors for MGN using well scrutinized methods together with expanding the inclusion criteria to patients with gastric dysplasia. As a result, moderate or severe IM in the gastric body and family history of gastric cancer were independent risk factors for MGN after adjusting for other variables.

IM is an established strongest risk factor for gastric cancer. An epidemiological study suggested that patients with IM have more than a 10-fold increased risk of developing gastric cancer, ${ }^{21}$ and our previous findings were similar, i.e., a 10.9-fold risk. ${ }^{22}$ In addition, the odds ratio of gastric cancer was found to be 29.3 for patients with severe IM at a 5-year follow-up. ${ }^{23}$ IM tends to appear first at the incisura angularis and extends to the neighboring mucosa in both the antrum and body. Therefore, the severity of IM in the gastric body rather than in the antrum reflects the risk of gastric cancer more exactly. ${ }^{24}$ The results of our study, that moderate or severe IM not in the antrum but in the corpus was independent risk factor for MGN further corroborate the fact.

Even though many studies have evaluated risk factors for MGC, studies that properly addressed the relationship between IM and MGC are surprisingly scarce. Recently, a large retrospective cohort study suggested that IM was not a risk factor for MGC in patients who underwent ER of gastric neoplasm including low-grade dysplasia. ${ }^{25}$ However, the proportion of the patients with IM was unexpectedly high (98.2\%), and because $95.0 \%$ of IM was severe metaplasia, evaluation according to the 
severity of IM was not possible. Another Korean study which evaluated risk factors for synchronous and metachronous gastric neoplasm in patients who underwent endoscopic submucosal dissection of gastric neoplasm suggested that old age ( $>65$ years) was the only independent risk factor of multiple gastric neoplasm. ${ }^{26}$ However, in this study, IM was classified as simply positive or negative and the mean follow-up period was too short (16 months). In addition, other studies which suggested old age as an independent risk factor of MGC did not evaluate severity of IM in the background gastric mucosa.,27 Therefore, considering that the prevalence of IM increases with age, ${ }^{28}$ there is a possibility that age might be a confounding factor and IM was a true risk factor of MGC in these studies.

In contrast with age, family history of gastric cancer was an independent risk factor for MGN after adjusting for other variables in the present study. These results are consistent with previous studies, ${ }^{5,29}$ and imply that family history of gastric cancer increases the susceptibility to gastric carcinogenesis and field cancerization irrespective of other factors like $H$. pylori infection and IM. ${ }^{30,31}$

Several earlier studies reported that gastric atrophy is risk factor of MGC after ER of EGC. ${ }^{3,27}$ In the present study, we evaluated the severity of gastric atrophy by serum PG test for two reasons: the endoscopic visual evaluation is subjective with high interobserver variability, ${ }^{32}$ and histology has a possibility of sampling errors and is sometimes not-available for evaluation of gastric atrophy. However, unlike IM, gastric atrophy was not an independent risk factor of MGN.

Little is known regarding the relationship between aberrant DNA methylation and the risk of MGN. In the present study, we compared the methylation level of several candidate genes in 19 patients who developed MGN with age- and sex- matched patients without MGN. We found that only the methylation level of MOS was significantly elevated in patients with MGN as compared to those without MGN. These results are consistent with our previous studies which suggested that MOS may be used as a surrogate marker for gastric cancer risk, since the methylation level of MOS is related with severity of IM and more importantly, hypermethylation of MOS persists after suppression of $H$. pylori infection. ${ }^{11-13}$ The mechanism of epigenetic carcinogenesis through aberrant DNA methylation is usually explained by silencing of tumor suppressor gene resulting from inactivation of promoter regions. Therefore, the relationship between hypermethylation of MOS which is a proto-oncogene and increased risk of MGN could be considered unreasonable. However, this paradoxical phenomenon observed in our study implies that MOS is a passenger gene rather than a driver gene. Because methylation of driver genes which are directly involved in the gastric carcinogenesis occurs only in a very small fraction of cells, the methylation level of drive genes is very low. ${ }^{6}$ In contrast, although passenger genes are unlikely to be causally involved in the gastric carcinogenesis, the methylation level of these genes is high and more apt to be clinically measured. ${ }^{33}$ Therefore, to evaluate the degree of epigenetic field defects which reflect risk of gastric neoplasm including dysplasia, these passenger genes could be more promising surrogate markers than driver genes. In the present study, we demonstrated that MOS could be a candidate molecular marker predicting MGN in the patients who underwent ER of gastric neoplasm. Because our study clearly implies that patients who have IM and show high methylation level of MOS have a high risk of MGN, more intensive surveillance should be performed in these patients. In future, it would be expected that if we discover and combine more biomarkers for risk of MGN, we could narrow the group who needs more intensive surveillance after ER of gastric neoplasm. For example, we are considering combining aberrant methylation of MOS and the expression of CDX2, whose levels correlated with the IM grade in the gastric body ${ }^{34,35}$ to predict risk of MGN.

This study has a limitation in that we could not perform methylation-specific PCR in all patients. Therefore, in order to draw definite conclusions, further study including a higher number of subjects might be needed. In addition, because this study was performed in a geographically restrained population, caution should be taken regarding the conclusions extrapolated in terms of impacting global clinical practice.

In conclusion, in the patients who underwent ER of gastric neoplasm, moderate or severe corpus IM and family history of gastric cancer were independent risk factors for MGN and the methylation level of MOS was significantly higher in patients who developed MGN than in those who did not. Therefore, more intensive endoscopic surveillance should be performed in individuals with family history of gastric cancer and moderate to severe corpus IM, and additionally show high methylation level of MOS even after ER of gastric neoplasm.

\section{CONFLICTS OF INTEREST}

No potential conflict of interest relevant to this article was reported.

\section{ACKNOWLEDGEMENTS}

This work was supported by the National Research Foundation of Korea (NRF) grant for the Global Core Research Center (GCRC) funded by the Korea government (MSIP) (number: 20110030001).

\section{REFERENCES}

1. Nakajima T, Oda I, Gotoda T, et al. Metachronous gastric cancers after endoscopic resection: how effective is annual endoscopic surveillance? Gastric Cancer 2006;9:93-98.

2. Kato M, Nishida T, Yamamoto K, et al. Scheduled endoscopic sur- 
veillance controls secondary cancer after curative endoscopic resection for early gastric cancer: a multicentre retrospective cohort study by Osaka University ESD study group. Gut 2013;62:14251432.

3. Hanaoka N, Uedo N, Shiotani A, et al. Autofluorescence imaging for predicting development of metachronous gastric cancer after Helicobacter pylori eradication. J Gastroenterol Hepatol 2010;25: 1844-1849.

4. Watari J, Moriichi K, Tanabe H, et al. Biomarkers predicting development of metachronous gastric cancer after endoscopic resection: an analysis of molecular pathology of Helicobacter pylori eradication. Int J Cancer 2012;130:2349-2358.

5. Kim YI, Choi IJ, Kook MC, et al. The association between Helicobacter pylori status and incidence of metachronous gastric cancer after endoscopic resection of early gastric cancer. Helicobacter 2014;19:194-201.

6. Ushijima T, Hattori N. Molecular pathways: involvement of Helicobacter pylori-triggered inflammation in the formation of an epigenetic field defect, and its usefulness as cancer risk and exposure markers. Clin Cancer Res 2012;18:923-929.

7. Chiba T, Marusawa H, Ushijima T. Inflammation-associated cancer development in digestive organs: mechanisms and roles for genetic and epigenetic modulation. Gastroenterology 2012;143:550563.

8. Graham DY. Helicobacter pylori eradication and metachronous gastric cancer. Clin Gastroenterol Hepatol 2014;12:801-803.

9. Yoon H, Kim N, Lee HS, et al. Effect of endoscopic screening at 1-year intervals on the clinicopathologic characteristics and treatment of gastric cancer in South Korea. J Gastroenterol Hepatol 2012;27:928-934.

10. Shin CM, Kim N, Jung Y, et al. Role of Helicobacter pylori infection in aberrant DNA methylation along multistep gastric carcinogenesis. Cancer Sci 2010;101:1337-1346.

11. Shin CM, Kim N, Jung Y, et al. Genome-wide DNA methylation profiles in noncancerous gastric mucosae with regard to Helicobacter pylori infection and the presence of gastric cancer. Helicobacter 2011;16:179-188.

12. Shin CM, Kim N, Park JH, et al. Prediction of the risk for gastric cancer using candidate methylation markers in the non-neoplastic gastric mucosae. J Pathol 2012;226:654-665.

13. Shin CM, Kim N, Lee HS, et al. Changes in aberrant DNA methylation after Helicobacter pylori eradication: a long-term follow-up study. Int J Cancer 2013;133:2034-2042.

14. Soetikno R, Kaltenbach T, Yeh R, Gotoda T. Endoscopic mucosal resection for early cancers of the upper gastrointestinal tract. J Clin Oncol 2005;23:4490-4498.

15. Shin CM, Kim N, Lee HS, et al. Validation of diagnostic tests for Helicobacter pylori with regard to grade of atrophic gastritis and/ or intestinal metaplasia. Helicobacter 2009;14:512-519.

16. Yoon H, Kim N, Lee HS, et al. Helicobacter pylori-negative gastric cancer in South Korea: incidence and clinicopathologic characteristics. Helicobacter 2011;16:382-388.
17. Miki K, Ichinose M, Kawamura N, et al. The significance of low serum pepsinogen levels to detect stomach cancer associated with extensive chronic gastritis in Japanese subjects. Jpn J Cancer Res 1989;80:111-114.

18. Dixon MF, Genta RM, Yardley JH, Correa P. Classification and grading of gastritis: the updated Sydney System. International workshop on the histopathology of gastritis, Houston 1994. Am J Surg Pathol 1996;20:1161-1181.

19. Yoon H, Kim SG, Choi J, et al. Risk factors of residual or recurrent tumor in patients with a tumor-positive resection margin after endoscopic resection of early gastric cancer. Surg Endosc 2013;27: 1561-1568.

20. Herman JG, Graff JR, Myohanen S, Nelkin BD, Baylin SB. Methylation-specific PCR: a novel PCR assay for methylation status of CpG islands. Proc Natl Acad Sci U S A 1996;93:9821-9826.

21. Leung WK, Sung JJ. Review article: intestinal metaplasia and gastric carcinogenesis. Aliment Pharmacol Ther 2002;16:1209-1216.

22. Kim N, Park RY, Cho SI, et al. Helicobacter pylori infection and development of gastric cancer in Korea: long-term follow-up. J Clin Gastroenterol 2008;42:448-454.

23. You WC, Li JY, Blot WJ, et al. Evolution of precancerous lesions in a rural Chinese population at high risk of gastric cancer. Int J Cancer 1999;83:615-619.

24. Cassaro M, Rugge M, Gutierrez 0, Leandro G, Graham DY, Genta RM. Topographic patterns of intestinal metaplasia and gastric cancer. Am J Gastroenterol 2000;95:1431-1438.

25. Bae SE, Jung HY, Kang J, et al. Effect of Helicobacter pylori eradication on metachronous recurrence after endoscopic resection of gastric neoplasm. Am J Gastroenterol 2014;109:60-67.

26. Jang MY, Cho JW, Oh WG, et al. Clinicopathological characteristics of synchronous and metachronous gastric neoplasms after endoscopic submucosal dissection. Korean J Intern Med 2013;28: 687-693.

27. Han JS, Jang JS, Choi SR, et al. A study of metachronous cancer after endoscopic resection of early gastric cancer. Scand J Gastroenterol 2011;46:1099-1104.

28. Kim N, Park YS, Cho SI, et al. Prevalence and risk factors of atrophic gastritis and intestinal metaplasia in a Korean population without significant gastroduodenal disease. Helicobacter 2008;13: 245-255.

29. Lee IS, Park YS, Kim KC, et al. Multiple synchronous early gastric cancers: high-risk group and proper management. Surg Oncol 2012;21:269-273.

30. Shin CM, Kim N, Yang HJ, et al. Stomach cancer risk in gastric cancer relatives: interaction between Helicobacter pylori infection and family history of gastric cancer for the risk of stomach cancer. J Clin Gastroenterol 2010;44:e34-e39.

31. Brenner H, Arndt V, Sturmer T, Stegmaier C, Ziegler H, Dhom G. Individual and joint contribution of family history and Helicobacter pylori infection to the risk of gastric carcinoma. Cancer 2000;88:274-279.

32. Eshmuratov A, Nah JC, Kim N, et al. The correlation of endoscopic 
and histological diagnosis of gastric atrophy. Dig Dis Sci 2010; 55:1364-1375.

33. Ushijima T. Epigenetic field for cancerization. J Biochem Mol Biol 2007;40:142-150.

34. Kang JM, Lee BH, Kim N, et al. CDX1 and CDX2 expression in in- testinal metaplasia, dysplasia and gastric cancer. J Korean Med Sci 2011;26:647-653.

35. Lee BH, Kim N, Lee HS, et al. The role of CDX2 in intestinal metaplasia evaluated using immunohistochemistry. Gut Liver 2012;6: 71-77. 